\title{
Evaluating Pediatric ACL Injury-Related Content Across Various Social Media Platforms
}

\author{
Anna Gussner'; Matt Rohde, BS²; Danika Baskar, BA²; Ted Ganley, $M D^{3}$; Kevin Shea, $M D^{2}$ \\ ${ }^{1}$ University of Utah, UT; ${ }^{2}$ Stanford University School of Medicine, CA; ${ }^{3}$ Children's Hospital of Philadelphia, PA \\ Correspondence to: Anna Gussner, 348 University Street E., Apt. 2, Salt Lake City, UT 84102, \\ E-mail: avgussner@gmail.com
}

Received: January 3, 2022; Accepted: January 9, 2022; Published: February 1, 2022

DOI: 10.55275/JPOSNA-2022-0008

\begin{abstract}
:
Background: Anterior Cruciate Ligament (ACL) tears are common in young athletes who rely on social media as a primary source of information. Evaluation of the information being shared across social media sites about pediatric ACL injuries has yet to be investigated. The purpose of this study was to investigate the content related to pediatric ACL tears across various social media platforms including Facebook, Instagram, YouTube, and TikTok.

Methods: The search terms "pediatric ACL," "pediatric ACL tear," and "ACL recovery" were used to identify relevant groups and postings. Each of these search terms was entered into each platform to find content with the highest number of followers, views, and/or posts. This content was then analyzed and categorized based on common themes of focus.

Results: Across four identified Facebook groups, there were over 13,000 members collectively with 950 posts shared from May 12, 2021, to June 12, 2021. Posts were shared by caregivers of adolescents searching for a community of support from those who have undergone similar injuries and treatments. Related Instagram hashtags revealed 1.5 million posts centered mainly on athletes returning to play after sustaining ACL injuries. The top two Instagram pages together had more than 42,600 followers focused on successful recovery anecdotes. The top five YouTube videos total 523,000 views and share educational content, surgical techniques, and patient stories. The top four TikTok hashtags have over 64 million total views with videos about personal experiences before and after ACL surgery.

Conclusion: Our findings revealed widespread sharing of anecdotal experiences after ACL injury. The advice shared may be valuable from a community support perspective. The relatively limited physician presence and monitoring of these social media sites may compromise the quality of medical information. Providers caring for pediatric athletes may benefit from increased knowledge about social media resources widely used by young athletes. Physicians may consider novel approaches to monitoring these sites and offering higher-quality information to patients and families to support better decision-making.
\end{abstract}


Level of Evidence: Level IV: Case series (no historical or control group)

\section{Key Concepts:}

- Anterior Cruciate Ligament (ACL) tears are common in young athletes who rely on social media as a primary source of information.

- Patients are turning to resources such as Facebook, YouTube, Instagram, and TikTok to find a community of support, ask for second opinions, receive advice, and discover medical information.

- Physicians may consider involvement on social media platforms to provide accurate medical information from an educational standpoint and support patients and their caregivers in making informed decisions.

\section{Introduction}

In the last decade, the internet and social media have gained significant influence on the population and have begun to change the way people access and validate information; this also applies to how people are accessing medical information. ${ }^{1-3}$ Not only have patients and caregivers increased their usage of online resources to access medical information, but they have also considerably increased their confidence in the information they find online. ${ }^{4}$

Healthcare providers must now be aware that their patients are turning to resources such as Facebook, YouTube, Instagram, and TikTok to find a community of support, ask for second opinions, receive advice, and discover medical information. As with many forms of technology, the use of social media to access medical information can be a benefit or a hindrance. Much of the information being accessed through these sources is created and distributed by patients and their families based on personal experiences, often with no physician oversight. Crowdsourcing, or gaining information and ideas from large groups of people often through internet resources, has become an increasingly common way for patients to utilize social media platforms to seek medical advice. $^{5}$

There is no shortage of information shared online about sports, related injuries, and treatments. This includes content on pediatric ACL cases with advice generally shared based on a user's personal experiences with an injury and subsequent recovery. A study published in 2017 found that $93.4 \%$ of the Instagram posts with the hashtag \#aclsurgery posted over a 6-month period were postoperative photos documenting a personal recovery story. ${ }^{6}$ When shared appropriately and reviewed by certified medical professionals involved in the treatment of pediatric ACL injury, this information has the potential to be extremely valuable to the public. However, as the accuracy of medical information shared online is usually not reviewed by a physician, this can lead to misinformation about options for care. This emphasizes the need for providers to be aware of this content across online platforms to best advise their patients who may be accessing these sources. These platforms may be a valuable resource for physicians to monitor trends and patient/family concerns about conditions and help improve patient-centered decision-making skills.

Patients and their families increasingly rely on alternative sources from the internet to gain access to medical information because of social media accessibility. The purpose of this study is to better understand who is sharing pediatric ACL content as well as the amount of content related to pediatric ACL injuries across social media platforms.

\section{Materials and Methods}

The social media platforms Facebook, Instagram, YouTube, and TikTok were searched using the terms 
"Pediatric ACL," "Pediatric ACL Tear," and "ACL Recovery." The search terms were chosen with the intent to be as broad as possible to query platforms for related content.

The top four Facebook groups with the highest number of followers as of June 12, 2021, were recorded, and the number of posts for these groups was recorded for the time between May 12 to June 12, 2021. The top five Instagram hashtags with the most posts and the top two Instagram accounts with the most followers as of June 12, 2021, were recorded. The top four TikTok hashtags with the highest number of views were recorded on June 19, 2021, and the top five YouTube videos with the most views were recorded on June 22, 2021. Different numbers of each category and each platform were reviewed based on how many posts, views, or followers they had. For Facebook groups, groups with over 500 members were reviewed. The top two Instagram accounts each had over 20,000 followers and were the only accounts looking at community outreach and support. The next largest Instagram accounts were personal accounts with significantly fewer followers.

Content across all platforms was analyzed quantitatively to identify the reach of related content that was shared by recording view count and number of followers for groups/pages of interest. Additionally, shared content was also classified into thematic categories to study focused areas of interest and discussion surrounding pediatric ACL injury.

\section{Results}

The Facebook search of the term "Pediatric ACL" revealed four prominent groups with content relating to ACL tears. These groups collectively have over 13,000 members and 950 posts. The groups were primarily run by caregivers who use the platform to find a community of support from others who have been through similar injuries or treatments. Many of the posts shared by the caregivers were referencing their own experiences and opinions to answer other caregivers' questions about treatment options or second opinions. All of the top four groups were private, and none of them have an active physician presence in the group. This was determined by searching the members for Dr., M.D., or D.O. mentioned in their name, bio, or profile. The most active group, "I survived ACL surgery" had 6,200 members as of June 12, 2021, and had 461 new postings in the 1-month period between May 12, 2021, and June 12, 2021.

The top five Instagram hashtags were \#ACL, \#aclrecovery, \#aclsurgery, \#aclrehab, and \#tornacl (Figure 1). Hashtags on Instagram are used to categorize posts of similar content. Since Instagram went online in 2010, these hashtags have accumulated a collective 1.5 million posts that focus on athletes returning to play after an ACL injury. When compared to clubfoot, another relatively common childhood condition, the top Instagram hashtag \#ACL had 930,915 posts and the top clubfoot hashtag \#clubfoot had 59,400+ posts when it was observed on April 15, 2021. ${ }^{7}$ The top two Instagram accounts focused on Pediatric ACL injuries were @ theaclclub and @acl_recovery_club,with 21,800 and 20,800 followers, respectively.

The five YouTube videos with the most views totaled over 523,000 views over the past 4 to 9 years that the videos have been posted, and focused on educational content, surgical techniques, and sharing patient stories (Figure 2). Three of the top five videos were posted by Boston Children's Hospital and all five videos had an individual physician's or hospital's input.

The top four TikTok hashtags totaled over 64 million views since TikTok's release in 2016 and focused on a patient's condition pre- and post-surgery. These hashtags were \#aclsurgery with 30.8 million views, \#aclrecovery with 13.8 million views, \#acltear with 12 million views, and \#aclrehab with 4.4 million views.

It is important to note that not all of the findings in these searches were explicitly pediatric based. However, the primarily young follower base of Instagram, YouTube, and TikTok gave way to a high yield of pediatric postings and ACL injury cases. On Facebook, the community support groups were generally parents or caregivers of children who were dealing with an ACL injury. 


\begin{tabular}{c|c|c|}
\hline Platform & Hashtag & Number of Posts on 6/20/21 \\
\hline Instagram & \#ACL & 930,915 \\
& \#aclrecovery & 267,909 \\
\hline & \#aclsurgery & 134,822 \\
\hline Platform & \#aclrehab & 122,218 \\
\hline TikTok & Hashtag & 42,402 \\
\hline & \#aclsurgery & Number of Views on $6 / \mathbf{1 9 / 2 1}$ \\
\hline & \#aclrecovery & 30.8 million \\
\hline & \#acltear & 13.8 million \\
\hline & \#aclrehab & 12 million \\
\hline
\end{tabular}

Figure 1. Top ACL-related hashtags on Instagram and TikTok.

Name of YouTube Video

Views on $6 / 22 / 21$

ACL injury - Stephanie's story - Boston Children's Hospital 203k

ACL injury treatment at Boston Children's Hospital $179 \mathrm{k}$

ACL injuries | Q\&A with Dr. Jay Lee

$83 \mathrm{k}$

\section{Surgical Reduction and Fixation of Tibial Spine Fractures in Children 30k Overcoming an ACL Injury - Olympic Bound Slopestyle Skier \\ $28 \mathrm{k}$}

Figure 2. Related YouTube videos with highest views.

\section{Discussion}

This work demonstrates that patients/families use social media platforms for interaction about ACL injury. Facebook, Instagram, and TikTok did not have a substantial physician presence to oversee or check the accuracy of the medical information being provided. On Facebook, where the groups primarily focused on finding a support community, the majority of the followers and actively posting participants were caregivers of children and young adults with an ACL injury. ${ }^{8}$ They gave recommendations and advice for treatment options based on their own experiences and opinions. This is concerning for the reliability and validity of the information being dispersed. YouTube was the only source analyzed where physicians were actively involved in the output and distribution of medical information. Our findings follow the trends found by similar studies looking at the social media presence of different medical conditions. Clubfoot, for example, which is the most common congenital foot defect in children, has seen a large growth in the use of social media as an avenue for caregivers to receive advice from their peers. ${ }^{9}$ 
Involvement has been climbing and more people have high levels of confidence in the accuracy of the medical information they find through social media sources. ${ }^{4}$

The involvement of the caregivers as the main source of involvement and postings on the social media platforms also raises unique ethical and privacy concerns. Crowdsourcing, or obtaining input from large numbers of people, in the case of social media sites, raises the possible issues of privacy and consent for the personal health information being shared and then distributed on the internet. When the caregiver is posting about a minor's health condition, additional issues of consent can arise. ${ }^{10}$ Another problem that has been explored is the negative impact that misinformation can have on patients receiving appropriate and timely treatment. In a full-text analysis of 57 articles on misinformation in medicine, researchers from the United Kingdom and Italy found an increasing trend in medical misinformation between 2012 and 2018 and identified social media as a major propagator. ${ }^{11}$

For a physician, providing medical advice on specific cases through these social media platforms may not be viable. The nature of medical conditions, including ACL injuries, are often complex, with multiple factors. While individualized advice is impossible through social media channels, it may be more plausible for physicians to develop an online presence in these groups. This could include the presentation of higher-quality information as well as links to higher-quality sources of information. Additionally, patients could be encouraged to develop a high-quality, evidence-based treatment plan with their physician-specific to their injury.

\section{Limitations}

This research is subject to several limitations. The first being that Twitter (which has 186 million daily active users-38 million of which are in the United States) was not examined in this paper because it did not lend itself as cleanly to the simple quantitative analysis performed on the other platforms. ${ }^{12}$ The simple form of quantitative analysis used in our study does not provide sophisticated results compared to other social media study methods such as sentiment analysis. ${ }^{13-16}$ Using sentiment analysis could have provided a deeper insight into the content of the social media posts that were examined. Another limitation concerns the way credentials such as Dr., M.D., and D.O. in the names, bios, or profiles of personal social media accounts were analyzed. Surely, not all medical professionals include their credentials on social media. For the purposes of this study, use of these credentials was used as a proxy for physician presence in a social media group. If they did not list their credentials, we assumed they were not acting as a physician on the platform.

\section{Summary}

The communities formed and information shared on the social media platforms of Facebook, Instagram, YouTube, and TikTok function to provide support amongst patients and their caregivers dealing with pediatric ACL injuries. These support communities mainly share information and advice based on personal experiences, but there may be a limited amount of physician oversight and review of the quality and validity of the medical information being provided. The information posted on social media platforms may be valuable when functioning as emotional support to other people dealing with similar issues. Physicians caring for patients with Pediatric ACL injuries should be aware of the information coming from the social media platforms that are extensively used by this population. Physicians may consider involvement on these platforms to provide accurate medical information from an educational standpoint and support patients and their caregivers in making informed decisions.

\section{References}

1. Green B, Hope A. Promoting clinical competence using social media. Nurse Educ. 2010;35(3):127-129. doi: https://doi.org/10.1097/ NNE.0b013e3181d9502b.

2. Moorhead SA, Hazlett DE, Harrison L, et al. A new dimension of health care: systematic review of the uses, benefits, and limitations of social media for health communication. $J$ Med Internet Res. 2013;15(4):e85. doi: https://doi.org/10.2196/jmir.1933.

3. Heidelberger CA. Health Care Professionals' Use of Online Social Networks. Published online March 25, 2011. Available at: https:/cahdsu. wordpress.com/2011/04/07/infs-892-health-care-professionals-use-ofonline-social-networks/. Accessed December 15, 2021. 
4. Dalmer NK. Questioning reliability assessments of health information on social media. J Med Libr Assoc. 2017;105(1):61-68. doi: https://doi. org/10.5195/jmla.2017.108.

5. Hors-Fraile S, Atique S, Mayer MA, et al. The unintended consequences of social media in healthcare: new problems and new solutions. Yearb Med Inform. 2016;(1):47-52. doi: https://doi.org/10.15265/IY-2016-009.

6. Ramkumar PN, La TJ, Fisch E, et al. Integrating social media and anterior cruciate ligament surgery: an analysis of patient, surgeon, and hospital use. Arthroscopy. 2017;33(3):579-585. doi: https://doi.org/10.1016/j. arthro.2016.08.021

7. Tonkovich N, Baskar D, Frick S. The impact of the digital age and social media on connecting the clubfoot community. Cureus. 2021;13(7):e16780 doi: https://doi.org/10.7759/cureus.16780.

8. Grajales FJ 3rd, Sheps S, Ho K, et al. Social media: a review and tutorial of applications in medicine and health care. J Med Internet Res. 2014;16(2):e13. doi: https://doi.org/10.2196/jmir.2912.

9. Hanna G, Batko BD, Potter J, et al. The role of social media in clubfoot: information sharing and social support. J Child Orthop. 2021;15(1):81-88. doi: https://doi.org/10.1302/1863-2548.15.200176.

10. Denecke K, Bamidis P, Bond C, et al. Ethical issues of social media usage in healthcare. Yearb Med Inform. 2015;10(1):137-147. doi: https://doi. org/10.15265/IY-2015-001.
11. Wang Y, McKee M, Torbica A, et al. Systematic literature review on the spread of health-related misinformation on social media. Soc Sci Med 1982. 2019;240:112552. doi: https://doi.org/10.1016/j. socscimed.2019.112552.

12. Iqbal M. Twitter Revenue and Usage Statistics (2021). Published online November 12, 2021. Available at: https://www.businessofapps.com/data/ twitter-statistics/. Accessed January 2, 2022.

13. Dominy CL, Arvind V, Tang JE, et al. Scoliosis surgery in social media: a natural language processing approach to analyzing the online patient perspective. Spine Deform. 2021:1-8. Published online October 28, 2021. doi: https://doi.org/10.1007/s43390-021-00433-0.

14. Bozkurt AP, Aras I. Cleft lip and palate youtube videos: content usefulness and sentiment analysis. Cleft Palate Craniofac. 2021;58(3):362-368. doi: https://doi.org/10.1177/1055665620948722.

15. Rao SA, Ravi MS, Zhao JW, et al. Social media responses to elective surgery cancellations in the wake of COVID-19. Ann Surg. 2020;272(3):e246-e248. doi: https://doi.org/10.1097/ SLA.0000000000004106.

16. Workewych AM, Ciuffetelli Muzzi M, Jing R, et al. Twitter and traumatic brain injury: a content and sentiment analysis of tweets pertaining to sport-related brain injury. SAGE Open Med. 2017;5:2050312117720057. doi: https://doi.org/10.1177/2050312117720057. 